\title{
A Geographically Distinct Case of Fatal Methanol Toxicity from Ingestion of a Contaminated Hand Sanitizer Product During the COVID-19 Pandemic
}

\author{
Daniel L. Overbeek $^{1}$ (D) C C. James Watson ${ }^{1,2} \cdot$ Nicolette R Castañeda $^{2} \cdot$ Michael Ganetsky $^{1,2}$
}

Received: 29 September 2020 / Revised: 4 January 2021 / Accepted: 13 January 2021 / Published online: 2 February 2021

(C) American College of Medical Toxicology 2021

\begin{abstract}
The COVID-19 pandemic has triggered outbreaks of unanticipated toxicities, including methanol toxicity. Multiple methanol outbreaks have been described, including contaminated hand sanitizer in the southwest USA. In this case, we describe a fatal case of methanol toxicity from hand sanitizer ingestion, geographically separated from the outbreak in the southwest USA and prior to the announcement of nationwide warnings by the Food and Drug Administration (FDA). The product was identified as one later recalled by the FDA for methanol contamination. Additionally, the consumption in this case was related to a desire to conceal alcohol consumption from family members. This case of methanol toxicity should increase awareness of the ease of which contaminated products can be widely distributed and of the use of alternative ethanol-containing products to obscure relapse in alcohol use disorder.
\end{abstract}

Keywords Methanol $\cdot$ Hand sanitizer $\cdot$ Outbreak $\cdot$ COVID-19

\section{Introduction}

Humans have long suffered from the accidental consumption of methanol. Toxicity has been described as far back as the late 1800s [1], frequently from consumption of home-distilled alcohols [2]. Methanol is a byproduct of the fermentation of alcoholic spirits, and individuals may be exposed when they drink alcohol which has not had the methanol component effectively removed. Methanol toxicity is marked by anion gap metabolic acidosis - which may be fatal - as well as retinal blindness [3]. During the COVID-19 pandemic, multiple outbreaks of methanol toxicity occurred. In Iran, where alcohol is largely prohibited, misinformation regarding the sterilizing qualities of alcoholic beverages resulted in the unregulated production of alcohol. Subsequently, an estimated 500

Supervising Editor: Andis Graudins, MB BS, PhD

Daniel L. Overbeek

daniel.overbeek@childrens.harvard.edu

1 Harvard Medical Toxicology Fellowship, Boston Children's Hospital, Boston, MA, USA

2 Department of Emergency Medicine, Beth Israel Deaconess Medical Center, Boston, MA, USA people have died from methanol exposure [4]. In the southwest USA, 15 cases of methanol toxicity have been linked to the ingestion of contaminated hand sanitizer products [5]. Herein, we describe a fatal case of methanol toxicity in June 2020 associated with the ingestion of hand sanitizer later linked to the aforementioned outbreak. This case is unique for its occurrence in a region remote from the published hand sanitizer poisonings and is notable because the ingestion occurred in the setting of trying to conceal an underlying alcohol use disorder.

\section{Case Report}

Our patient was a 27-year-old man who presented to a community emergency department in New England in June 2020 with vomiting and altered mental status. He had a history of alcohol use disorder with a recent rehabilitation admission, was living with his family, and drank hand sanitizer ("Blumen Clear Advanced Hand Sanitizer," distributed by 4e Brands North America, San Antonio, TX) at least twice over the prior 2 days, as he told his brother, reportedly attempting to hide his continued alcohol use from his parents. His parents reported that he previously had been known to drink 
alternative ethanol sources, and five empty hand sanitizer bottles $(221 \mathrm{~mL}$ each) were found near the patient, with another full bottle in the room, all of the same brand. The hand sanitizer (Fig. 1) listed 70\% ethanol as the only active ingredient; however, this hand sanitizer brand was later recalled by the FDA for contamination with methanol. The patient had been playing golf the morning of presentation and developed abdominal pain, vomiting, and blurry vision. Shortly after presenting to the emergency department, he became unresponsive, had multiple seizures, and developed cardiac arrest. He was resuscitated with advanced cardiovascular life support (ACLS) protocols including epinephrine and sodium bicarbonate boluses. He remained in profound shock necessitating multiple vasoactive agents and was transferred to a tertiary care hospital.

At the receiving center, laboratory studies showed a $\mathrm{pH}$ of 6.53, sodium $150 \mathrm{mEq} / \mathrm{L}$ (reference range $135-147 \mathrm{mEq} / \mathrm{L}$ ), potassium $6.4 \mathrm{mEq} / \mathrm{L}(3.5-5.4 \mathrm{mEq} / \mathrm{L})$, chloride $114 \mathrm{mEq} / \mathrm{L}$ (96-108 $\mathrm{mEq} / \mathrm{L})$, bicarbonate $5 \mathrm{mEq} / \mathrm{L}(22-32 \mathrm{mEq} / \mathrm{L})$, blood urea nitrogen $13 \mathrm{mg} / \mathrm{dL}(6-20 \mathrm{mg} / \mathrm{dL})$, creatinine 1.9 $\mathrm{mg} / \mathrm{dL}(0.5-1.2 \mathrm{mg} / \mathrm{dL})$, and glucose $83 \mathrm{mg} / \mathrm{dL}(70-100 \mathrm{mg} /$ $\mathrm{dL}$ ), with an anion gap of $31 \mathrm{mEq} / \mathrm{L}$. Serum ethanol concentration was undetectable, and serum osmolarity was 427 $\mathrm{mOsm} / \mathrm{kg}$, with a calculated osmolar gap of $118 \mathrm{mOsm} / \mathrm{kg}$. The patient was continued on vasoactive agents and was treated with fomepizole and bicarbonate as well as thiamine, folinic acid, and pyridoxine. Emergent renal replacement therapy was initiated within 4 hours of arrival to the receiving center. Continuous veno-venous hemodiafiltration was utilized instead of intermittent hemodialysis due to the patient's hypotension. A serum methanol concentration obtained on the day of presentation resulted at $240 \mathrm{mg} / \mathrm{dL}$, repeated on day 3 was $15, \mathrm{mg} / \mathrm{dL}$ and was undetectable by hospital day 4 .
The clinical presentation of severe metabolic acidosis was not consistent with ethanol intoxication, which triggered the deeper diagnostic investigation. Since this case occurred prior to the widespread FDA warning regarding methanol contaminated hand sanitizer during the COVID-19 pandemic and remote from the reports of cases in the southwest, the hand sanitizer was not immediately identified as the source of the methanol, but no other methanol sources were identified. The patient did not have a history of making or consuming homemade ethanol products, and no other possible methanol containing products were found in the home. This includes no methanol containing automotive chemicals and no homemade ethanol products or distillation equipment.

There was concern for severe neurologic injury, and the patient underwent post-arrest targeted temperature management. After the completion of temperature management and the confirmation of undetectable methanol concentrations, the patient was evaluated by the neurology service. On hospital day 9 , the clinical exam and confirmatory technetium-99 cerebral perfusion testing were both consistent with brain death. The patient was pronounced dead on hospital day 9 , and at the family's request, he underwent organ retrieval for donation. Consent for publication of this case was obtained and provided to the journal in accordance with JMT policy.

\section{Discussion}

Methanol toxicity is caused primarily by its metabolites. Methanol is metabolized via alcohol dehydrogenase (ADH) and aldehyde dehydrogenase to formaldehyde and formic acid, respectively. Formic acid accumulation as well as direct inhibition of cytochrome oxidase and mitochondrial respiration results in an elevated anion gap metabolic acidosis. The
Fig. 1 Hand sanitizer bottle found with the patient, and this brand was recalled by the FDA for methanol contamination.
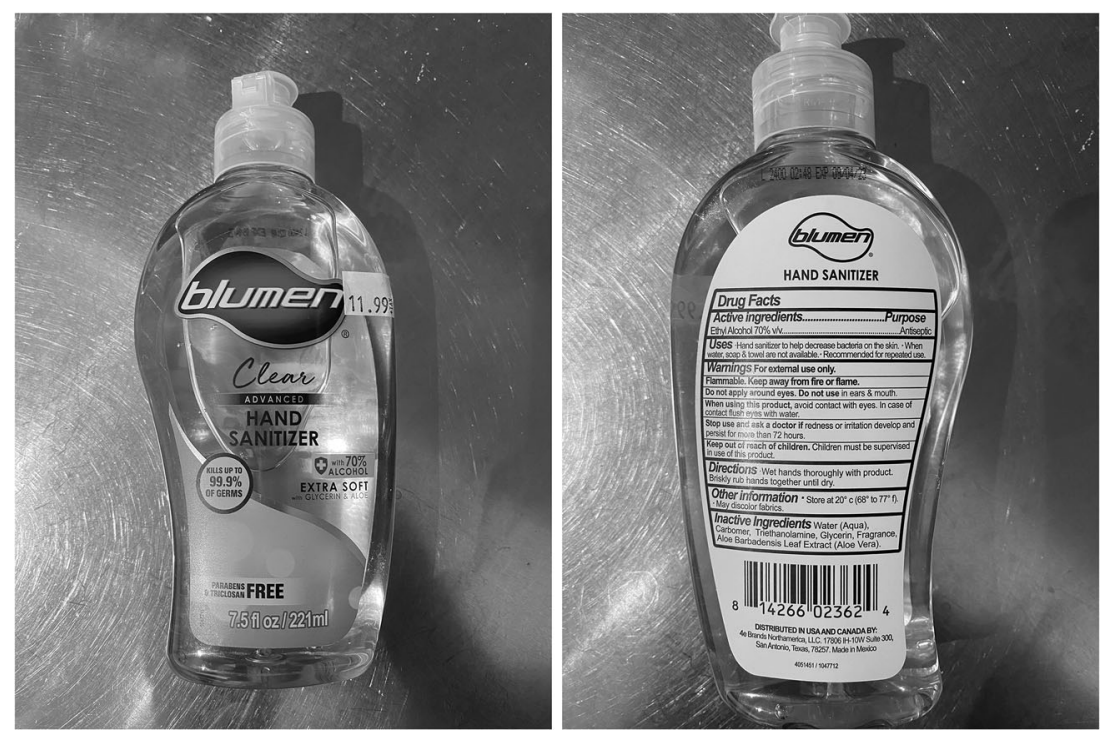
metabolic acidosis, if severe, can cause cardiovascular collapse. Formic acid is also a direct retinal toxin, which can cause vision loss [6].

The identification and diagnosis of methanol toxicity can be difficult, especially without a reported methanol ingestion. Initial presenting symptoms can be widely varied and nonspecific, including altered mental status, vomiting, abdominal pain, and vision changes. Patients may appear intoxicated, although methanol is not as inebriating as ethanol or ethylene glycol, and severely altered mental status should raise concern for comorbid processes such as coingestants, seizures, acidosis, cerebral edema, or putamenal hemorrhages [7]. The visual changes secondary to retinal toxicity are more specific but are not a sensitive finding [6].

Laboratory studies are critical to making the diagnosis: the classically described findings include acidemia from an elevated anion gap metabolic acidosis as well as an elevated osmolar gap. Methanol serum concentration is the gold standard for diagnosis, but these results can be delayed. Therefore, management is often initiated based on the presence of an anion gap metabolic acidosis, an elevated osmolar gap, or sufficient clinical suspicion.

Initial management of methanol toxicity includes inhibition of $\mathrm{ADH}$ to decrease the conversion of methanol into formaldehyde and formic acid, via ethanol or fomepizole (4methylpyrazole). Ethanol has been described as early as 1953 as a treatment for methanol intoxication and may be the only option if fomepizole is not available [8]. Fomepizole received FDA approval in the USA in 1997, has largely supplanted ethanol in the management of methanol and ethylene glycol toxicity, and is well tolerated with minimal adverse effects. Renal replacement therapies such as hemodialysis can readily clear methanol and its toxic metabolites, as well as correct the associated metabolic acidosis.

During the COVID-19 pandemic, there have been multiple outbreaks of methanol toxicity. In Iran, ethanol intake was touted to prevent or treat COVID-19. Given the historically limited access to ethanol, many Iranians purchased illicit ethanol products or attempted to produce their own ethanol. Tragically, this resulted in over 3000 cases of methanol toxicity reported in Iran in 2020 with over 500 confirmed deaths [9].

There was also a cluster of methanol exposures in the USA during the COVID-19 pandemic. In May and June of 2020, there were reports of methanol toxicity related to hand sanitizer ingestion in Arizona and New Mexico, including at least 15 cases and 4 deaths. An initial warning was released by the United States Food and Drug Administration (FDA) on June 19, 2020, regarding nine hand sanitizer products from the manufacturer Eskbiochem [10]. By that time, methanol contamination of hand sanitizers was already more widespread than realized. The hand sanitizer ingested in this case report was eventually added to the FDA warning almost 1 month after the patient presented [11].
This case highlights the tragic consequences of secondary public health crises associated with the COVID-19 pandemic, such as increases in substance use and limiting resources for treatment of substance use disorders [12]. The ingestion of hand sanitizer products for their ethanol content was common even preceding the pandemic, oftentimes in adolescents with exploratory ingestions or in patients with alcohol use disorder who have diminished access to traditional sources of ethanol [13]. Unfortunately, as manufacturing of cleaning supplies was rapidly scaled up to meet demand during the pandemic, there were likely consequent quality assurance lapses increasing risk of contamination, such as with methanol. The FDA recommends production standards for hand sanitizer, including "use[ing] the most accurate method of analysis available at the site for verification of ethanol content in a sample before each batch is released for distribution" [14]. Ironically, the rising consumer price of hand sanitizer during the pandemic likely dissuaded ingesting hand sanitizer as an alternate inebriating agent, which unfortunately did not occur in our case.

In mid-June 2020, the FDA issued a warning regarding methanol contamination of hand sanitizer products leading some companies to issue recalls shortly thereafter. The FDA also restricted the import of a large number of products. However, some of these products remain on the market and publicly available, as seen in a newspaper article dated August 15,2020 , which inadvertently displays a recalled hand sanitizer product of the same brand being used at a northern New England inn ${ }^{15}$. One primary limitation of the study is that we do not have analytical confirmation that the hand sanitizer contained methanol. The full hand sanitizer bottle is not available, it does not appear that he consumed any sanitizer from that bottle, and the other bottle was completely empty. This adds some doubt regarding the possible source; however, other possible sources were investigated, and no other likely sources were identified.

Methanol intoxication is highly dangerous and outbreaks of poisoning are a secondary consequence linked to the COVID-19 pandemic. We call for aggressive public health interventions, including consumer and manufacturer education as well as increased regulation of production and improved testing for contamination. Currently, the FDA recommends testing each batch of active ingredient, ethanol or isopropyl alcohol, for methanol contamination, but does not have any quality controls to confirm this testing ${ }^{16}$. Additionally, public health agencies need to focus on treatment of substance use disorders that have been exacerbated by the limited resources and increasing social isolation caused by the pandemic. Medical toxicologists will continue to play an important role identifying potentially dangerous scenarios as in this case, as well as crafting guidelines and offering treatment. 
Sources of Funding None.

\section{Compliance with ethical standards}

Consent for Publication Consent for publication of this case was obtained and provided to the journal in accordance with JMT policy.

Conflicts of Interest None.

\section{References}

1. Wood CA, Buller F. Poisoning by wood alcohol: cases of death and blindness from columbian spirits and other methylated preparations. J Am Med Assoc. 1904;XLIII:972.

2. Ahmad K. Methanol-laced moonshine kills 140 in Kenya. Lancet. 2000;356:1911.

3. Barceloux DG, Bond GR, Krenzelok EP, Cooper H, Vale JA. American Academy of Clinical Toxicology practice guidelines on the treatment of methanol poisoning. J Toxicol Clin Toxicol. 2002;40:415-46.

4. Delirrad M, Mohammadi AB. New methanol poisoning outbreaks in Iran following COVID-19 pandemic. Alcohol Alcohol. 2020;55: 347-8.

5. Yip L, Bixler D, Brooks DE, et al. Serious adverse health events, including death, associated with ingesting alcohol-based hand sanitizers containing methanol - Arizona and New Mexico, May-June 2020. MMWR Morb Mortal Wkly Rep. 2020. https:// doi.org/10.15585/mmwr.mm6932e1.

6. Yang CS, Tsai WJ, Lirng JF. Ocular manifestations and MRI findings in a case of methanol poisoning [5]. Eye. 2005;19:806-9.

7. Patil N, Becker M, Ganetsky M. Toxic alcohols: not always a clearcut diagnosis. Emerg Med Pr. 2010.

8. Bennett IL, Cary FH, Mitchell GL, Cooper MN. Acute methyl alcohol poisoning: a review based on experiences in an outbreak of 323 cases. Med (United States). 1953. https://doi.org/10.1097/ 00005792-195312000-00002.

9. Shokoohi M, Nasiri N, Sharifi H, Baral S, Stranges S. A syndemic of COVID-19 and methanol poisoning in Iran: time for Iran to consider alcohol use as a public health challenge? Alcohol. 2020;87:25-7.

10. FDA. FDA Advises Consumers not to use hand sanitizer products manufactured by Eskbiochem. 2020. https://www.fda.gov/drugs/ drug-safety-and-availability/fda-advises-consumers-not-use-handsanitizer-products-manufactured-eskbiochem.

11. FDA. FDA updates on hand sanitizers consumers should not use.https://www.fda.gov/drugs/drug-safety-and-availability/fdaupdates-hand-sanitizers-consumers-should-not-use. Published 2020.

12. Ornell F, Moura HF, Scherer JN, Pechansky F, Kessler FHP, von Diemen L. The COVID-19 pandemic and its impact on substance use: implications for prevention and treatment. Psychiatry Res. 2020;289:113096.

13. Gormley NJ, Bronstein AC, Rasimas JJ, Pao M, Wratney AT, Sun $\mathrm{J}$, et al. The rising incidence of intentional ingestion of ethanolcontaining hand sanitizers. Crit Care Med. 2012;40:290-4.

14. US Department of Health and Human Services Food and Drug Administration. Temporary policy for manufacture of alcohol for incorporation into alcohol based hand sanitizer products during the public health emergency (COVID-19). 2020. https://www.fda.gov/ media/136390/download.

15. Fort L. August 15 update: The latest on the coronavirus and Maine. Bangor Daily News. https://bangordailynews.com/2020/08/15/ news/august-15-update-the-latest-on-the-coronavirus-and-maine/. Published August 15, 2020.

16. U.S. Department of Health and Human Services. Temporary policy for preparation of certain alcohol-based hand sanitizer products during the public health emergency (COVID-19) guidance for Industry. 2020. https://www.fda.gov/media/136289/download.

Publisher's Note Springer Nature remains neutral with regard to jurisdictional claims in published maps and institutional affiliations. 